Research Article

\title{
Optimal Atlas Segmentation on CT Images for Diagnosis of Pediatric Mycoplasma Pneumonia
}

\author{
Chunhong Cheng $\mathbb{D}^{D}$, Junyan Wang $\mathbb{D}$, Hongbo Li $\mathbb{i}$, and Liang Wang \\ Department of Radiology, Wuhan Sixth Hospital, Affiliated Hospital of Jianghan University, Wuhan 430015, Hubei, China \\ Correspondence should be addressed to Liang Wang; 189310007@stu.just.edu.cn
}

Received 21 June 2021; Accepted 22 July 2021; Published 29 July 2021

Academic Editor: Gustavo Ramirez

Copyright (C) 2021 Chunhong Cheng et al. This is an open access article distributed under the Creative Commons Attribution License, which permits unrestricted use, distribution, and reproduction in any medium, provided the original work is properly cited.

\begin{abstract}
This work aimed to explore the clinical application value of CT imaging technology based on the optimal Atlas segmentation algorithm (OASA) in the diagnosis of pediatric mycoplasma pneumonia (MP). Eighty-eight children with MP were selected and divided into group A (CT image based on the OASA) and group B (chest X-ray) according to the diagnosis methods. The detection rate, image feature performance, and image quality satisfaction of the two groups of children were compared. The results showed that the detection rate of group A was $97.73 \%$ and that of group B was $95.46 \%$, and there was no considerable difference between the two $(P>0.05)$. The pleural effusion detection rate of children in group A was evidently superior to that of X-ray group, while the increased bronchovascular shadows' detection rate was greatly inferior to that of X-ray group $(P<0.05)$. Comparison results of nodules' shadows, patchy shadows, acinar parenchyma shadows, and interstitial infiltration between two groups showed that there was no notable difference $(P>0.05)$. CT image quality satisfaction $(98.50 \%)$ was higher versus X-ray $(79.46 \%)(P<0.05)$. To sum up, CT images based on the OASA can be adopted in the clinical diagnosis of pediatric MP, and CT images were better than chest X-rays.
\end{abstract}

\section{Introduction}

Pediatric MP is usually an infectious respiratory disease caused by mycoplasma pneumonia $[1,2]$. The diseased population accounts for more than $50 \%$ of all children with pneumonia, and the incidence is increasing year by year. Pediatric MP will cause damage to various organs and bodily functions of children, which has a great impact on the normal growth and development of children [3]. The clinical symptoms of the child are mainly headache, fever, sore throat, and sputum, and the body fluids and sputum of the child are infectious. It is very easy to cause mycoplasma variant infection to occur, and it may cause death when it is more serious [4]. Since it lacks specific clinical symptoms, it is difficult to make an accurate diagnosis based on the symptoms and signs of the child. Mycoplasma pneumonia antibody detection is a common effective method for detecting pneumonia, and it has certain diagnostic value for pediatric MP [5]. However, its detection time is slightly longer, and it takes several days for the antibody titer to rise, and its wide application is limited. Therefore, finding a suitable diagnosis method is of great significance for the early prevention and subsequent treatment of MP.

With the rapid development of medical imaging technology, many kinds of imaging technology are utilized in the diagnosis of various clinical diseases, which provides more accurate information for doctors. CT and X-ray are effective methods for the clinical diagnosis of MP and they are widely adopted in the clinical diagnosis and treatment of pediatric MP $[6,7]$. In contrast to traditional X-ray, CT imaging technology has short scanning time, high resolution imaging, and high anatomical detail. It can observe the complex structure and find the small abnormal situation. CT imaging is currently the preferred method for detecting pulmonary diseases and subtle structural changes [8]. With the increasing adoption of CT technology in the medical field, image segmentation has become a research hotspot in medical image processing. Medical image segmentation is 
crucial for accurate disease positioning, 3D visualization, and subsequent treatment $[9,10]$. Due to the individual differences in the anatomical structure of lung tissue, there is also incomplete or low contrast of the lung fissure, which increases the difficulty of lung segmentation. Therefore, the study of lobar segmentation and its algorithm performance is of great significance for the clinical diagnosis of pulmonary diseases.

After the pulmonary anatomical structure was analyzed, the optimal Atlas map was utilized to search for the acute location of pulmonary fissure combined with the region of interest of pulmonary fissure. The CT image technology based on the OASA was proposed. The clinical adoption value of MP in diagnosing pediatric pneumonia by CT imaging technology based on the OASA was explored, which was compared with $\mathrm{X}$-ray diagnosis results. It was hoped that theoretical basis for the clinical diagnosis and subsequent treatment of pediatric MP could be provided.

\section{Materials and Methods}

2.1. Research Objects and Grouping. The 88 children with MP in the hospital from October 2018 to June 2020 were selected as the research objects. They were 47 males and 41 females. The children's age ranged from 6 months to 12 years, with an average age of $4.29 \pm 1.26$ (years). All patients were divided into CT group (group A) and X-ray group (group B) according to different diagnosis methods, with 44 cases in each group. This experiment had been approved by the ethics committee of the hospital, and the children and their families involved had been aware of and agreed with it.

Inclusion criteria were as follows: (I) patients met the diagnostic criteria of Pediatrics Mycoplasma Pneumonia in Practice; (II) patients were without congenital disease; (III) patients and their families had signed the informed consents; (IV) patients were without abnormalities in liver, kidney, heart, and lungs; and (V) patients were with complete clinical data. Exclusion criteria were as follows: (I) patients with chronic lung diseases, asthma, and so forth; (II) patients with mental or intellectual disabilities; (III) patients with abnormal immune function; and (IV) patients with severe infectious diseases.

2.2. Diagnosis Methods. The children in group A underwent CT examination using Philips Brilliance 16-slice spiral CT scanner. The children were placed in a supine position. The scanning range was from the tip of the lung to the bottom of the lung. The scanning time was $1 \mathrm{~s}$, the voltage was $120 \mathrm{kV}$, the current was $100-150 \mathrm{~mA}$, the layer thickness was $5 \mathrm{~mm}$, the layer distance was $5 \mathrm{~mm}$, and the pitch was 1.2 . The coronal and sagittal focal areas were reconstructed. After that, an enhanced scan was performed with iohexol as the contrast agent, and the injection speed was $1.5 \mathrm{~mL} / \mathrm{s}$. The distribution of lesions in the lungs of the children was observed, as well as the imaging characteristics of lung shadows and lung interstitium.

The children in group B took chest X-ray examination, Philips multifunction $\mathrm{X}$-ray machine was utilized to take the positive image of the chest of the children, and the chest was scanned in the conventional way. The scan parameters were set to voltage of $65 \mathrm{kV}$ and current of $0.6 \mathrm{mAs}$. The distribution of lesions in the lungs was observed, and so did the morphological characteristics, density of the lesions, and the morphological characteristics of the pulmonary mesenchyme and pleura.

2.3. CT Images Based on OASA. First, the lung area was extracted and positioned according to the CT image. The optimal threshold method [11] was utilized to identify and extract the lung area of the CT image, and then the lung area of interest was extracted. The noise in the area was mainly cleared and the boundary was smooth. The specific process was shown in Figure 1. follows:

The automatic update split threshold is calculated as

$$
G^{i+1}=\frac{U_{f}+U_{b}}{2}
$$

In equation (1), $U_{f}$ is the average gray value of the target area; $U_{b}$ is the average gray value of the background area; and $G^{i+1}$ is the segmentation threshold of step $i+1$.

The threshold is iteratively updated until the threshold in step $e$ no longer changes; that is, $G^{i+1}=G^{i}$.

Then, the initialization of the fissure area of the lung was carried out, which was mainly divided into three steps. the first step is pulmonary tracheal target area extraction. The air region of the CT image was selected as the seed point, the airway region was extracted by the $3 \mathrm{D}$ region growth method, and then the branches were refined one by one. Finally, the branches within the 15 nodes were taken as the final output of the pulmonary tracheal branches. The second step is target region segmentation of pulmonary vessels. The pulmonary vessels were segmented by fuzzy segmentation. The third step is calculation and regional initialization of the distance map of the pulmonary fissure area. 2D Euclidean distance transformation equation was adopted to calculate the distance from the pixel of lung tissue area to the "branch" of lung lobe, and the nearest pixel was divided into specific lung lobe area. The boundary between different lobe regions was the initial location of the lobe fissure. The Euclidean distance equation is expressed as follows:

$$
D=\sqrt{\left(x-x_{o}\right)^{2}+\left(y-y_{o}\right)^{2}} \text {. }
$$

In equation (2), $D$ is Euclidean distance; $(x o, y o)$ is the coordinate of the "branch" in the $2 \mathrm{D}$ image.

The Atlas segmentation scheme is mainly adopted to search the image with the most similar anatomical structure to the scanned image to be tested and to search the location of the lobe fissure according to the registration-segmentation technology, so as to complete the accurate segmentation of the lobe. (I) The template image that is closest to the location and characteristics of the pulmonary fissure is looked for; (II) registration transformation is performed to obtain the best mapping transformation parameters and transform mask 1 image into mask 2 image; and (III) fusion 


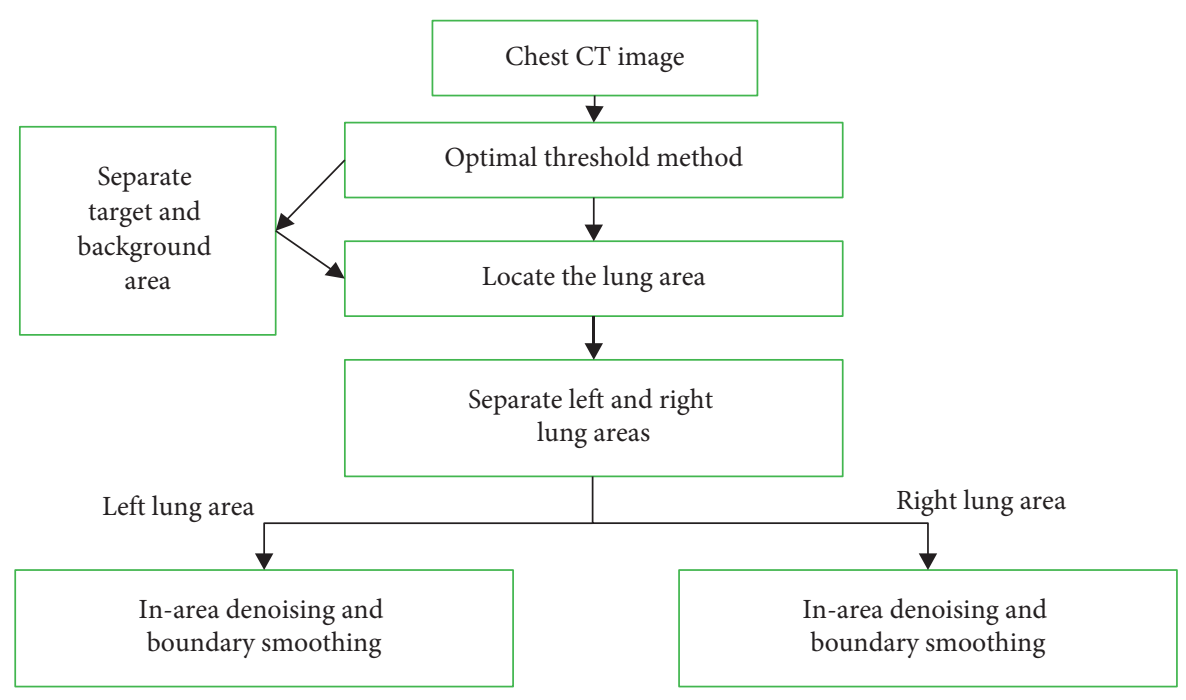

Figure 1: Lung area extraction process.

of mask 2 image and image to be tested is conducted to complete fissure detection. The specific process is shown in Figure 2.

The segmentation algorithm combines the prior knowledge of the map. The template map contains the original image and the mask image of the lung lobes. The location information of the lung lobes is represented by $P_{\text {landmark }}$, and the location information of the scanned image to be tested is represented by $q_{\text {landmark. The registration of the }}$ template image of the Atlas is based on the minimum deviation $\Delta$ of the two. The expression of the minimum deviation $\Delta$ is as follows:

$$
\Delta=\frac{1}{2}\left(\frac{p_{\text {Landmark }}(x 1, \ldots, x i)}{q_{\text {Landmark }}(x 1, \ldots, x i)}+\frac{p_{\text {Landmark }}(y 1, \ldots, y i)}{q_{\text {Landmark }}(y 1, \ldots, y i)}\right) .
$$

In equation (3), $\quad p_{\text {Landmark }}(x 1, \ldots, x i)$ and $p_{\text {Landmark }}(y 1, \ldots, y i)$ are the $x$ and $y$ coordinates of the lung lobes in the template image, respectively; and $q_{\text {Landmark }}(x 1, \ldots, x i)$ and $q_{\text {Landmark }}(y 1, \ldots, y i)$ are the $x$ and $y$ coordinates of the lung lobes in the image to be tested, respectively.

To enhance the calculation speed and segmentation accuracy, the minimum deviation combined with the perceptual hash matching method can be adopted to realize the fast search of the optimal Atlas for CT images. The calculation method of the optimal similarity $S(t)$ is as follows:

$$
S(t)=\sum_{t=1}^{n}\left(a K_{t}+b \Delta_{t}\right)
$$

In equation (4), $t$ is the registration template image; $K$ is the perceptual hash similarity; $K_{t}$ represents the hash similarity between the test image and the template image; $\Delta_{t}$ is the deviation value between the test image and the template image. $a$ is set to 0.25 and $b$ to 0.75 . If the value of $S(t)$ is greater than 0.85 , it is the optimal Atlas image.
The average distance MEAN, maximum distance MAX, and accuracy of area of interest $A_{\text {ROI }}$ of manually defined reference standard and automatic segmentation are adopted to evaluate the algorithm segmentation performance. The expression of the nearest voxel distance Dis for reference criteria and lobe segmentation results is as follows:

$$
\text { Dis }=\min \left\{\sqrt{\left(x_{A}-x_{m}\right)^{2}+\left(y_{A}-y_{M}\right)^{2}+\left(z_{A}-z_{M}\right)^{2}}\right\} \text {. }
$$

In equation (5), $\left(x_{A}, y_{A}, z_{A}\right)$ and $\left(x_{M}, y_{M}, z_{M}\right)$ represent the voxels defined by computer and manual segmentation, respectively.

$$
\begin{aligned}
\text { MEAN } & =\frac{\sum_{i=1}^{l} \sum_{j=1}^{m} \sum_{k=1}^{n} \operatorname{Dis}(i, j, k)}{l \times m \times n}, \\
\text { MAX } & =\max \{D i s\} .
\end{aligned}
$$

In equations (6) and (7), $l$ represents the size of volume data in the $x$ direction; $m$ is the size of volume data in the $y$ direction; and $n$ is the size of volume data in the $z$ direction. In addition, the standard deviation SD of voxel position is defined according to the mean distance value.

$$
A_{\mathrm{ROI}}=\frac{S_{A} \cap S_{M}}{S_{A} \cup S_{M}} \text {. }
$$

In equation (8), $A_{\mathrm{ROI}}$ represents segmentation accuracy. $S_{A}$ represents the area of the lung defined in $2 \mathrm{D}$ cross section. $S_{M}$ represents the area of the lung divided by a computer.

2.4. Observation Indexes. The clinical data of the two groups were compared, including age, gender, and onset time. The distribution of lesions (upper left lung lobe, lower left lung lobe, upper right lung lobe, middle right lung lobe, and lower right lung lobe), detection rate, and imaging characteristics were compared between the two groups. The satisfaction of 


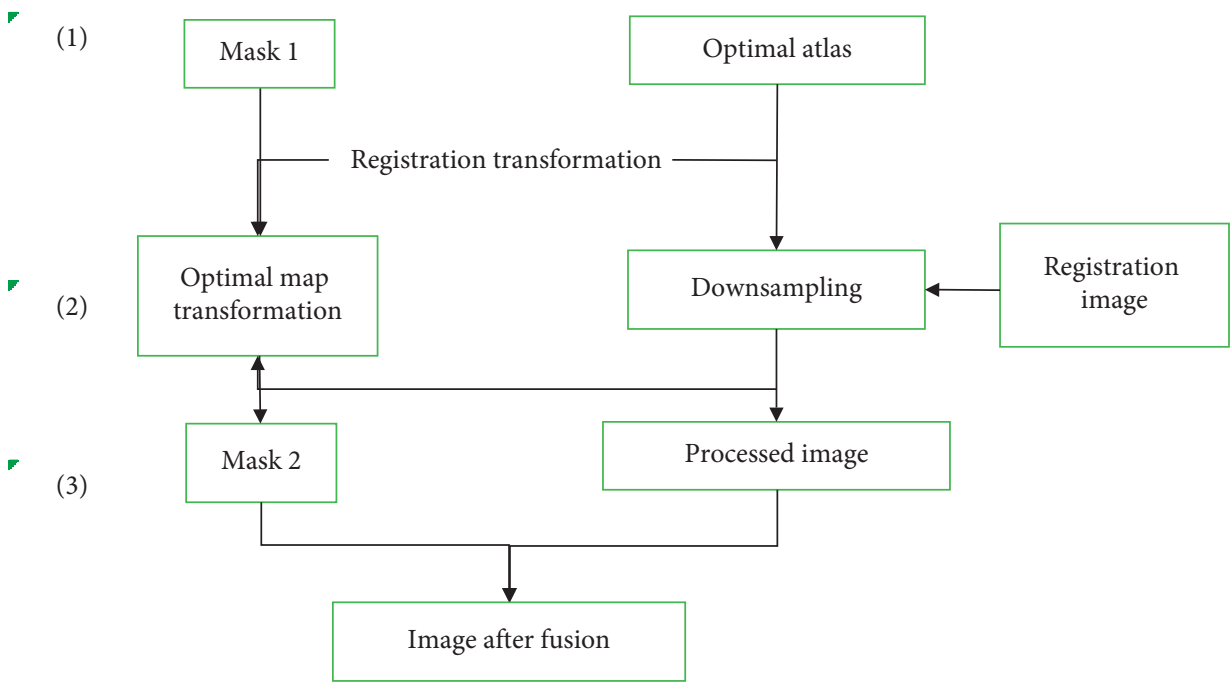

Figure 2: Flow of the OASA.

attending physicians with the image quality obtained by CT and X-ray examination was compared and analyzed.

2.5. Statistical Analysis. SPSS 20.0 statistical software was adopted for analysis. Measurement data were expressed as mean \pm standard deviation $(\bar{x} \pm s)$, and $t$-test was adopted for comparative analysis; count data were expressed as percentage (\%), and $\chi^{2}$ test was adopted for comparison and analysis between groups. If $P<0.05$, the difference was statistically significant.

\section{Results}

3.1. CT Image Segmentation Results Based on OASA. The OASA was utilized to segment the chest CT images, and the segmentation performances of the six parts including left upper lung lobe (ULL), left lower lung lobe (LLL), right upper lung lobe (URL), right middle lung lobe (MRL), and right lower lung lobe (LRL) were quantitatively evaluated using indicators of $A_{\mathrm{ROI}}$, MEAN, MAX, and SD. The detailed results are shown in Table 1 . According to the data, the OASA proposed in this work had strong robustness, and its MEAN, MAX, $A_{\mathrm{ROI}}$, and SD values were $9.22 \mathrm{~mm}$, $15.62 \mathrm{~mm}, 97.26 \%$, and 0.93 , respectively.

To further verify the segmentation effect of the OASA, it was compared with Elastix and NeftyReg segmentation methods. The performance comparison results of different segmentation algorithms are shown in Table 2. From the data, the MEAN, MAX, $A_{\mathrm{ROI}}$, and SD values of the Elastix method were $10.57 \mathrm{~mm}, 17.54 \mathrm{~mm}, 95.21 \%$, and 1.26 , respectively. The values of MEAN, MAX, $A_{\mathrm{ROI}}$, and SD of the NeftyReg method were $10.09,18.65,94.49 \%$, and 1.48 respectively. The values of MEAN, MAX, $A_{\mathrm{ROI}}$, and SD of the OASA were $9.22 \mathrm{~mm}, 15.62 \mathrm{~mm}, 97.26 \%$, and 0.93 , respectively. Based on the above results, the OASA proposed in this work was more accurate and more stable for the location of lung lobe region segmentation in CT images, and all performance indicators were superior to those of Elastix and NeftyReg segmentation methods.
TABle 1: Performance evaluation of the OASA.

\begin{tabular}{lcccccc}
\hline & \multicolumn{2}{c}{ Left lung } & \multicolumn{3}{c}{ Right lung } & \multirow{2}{*}{ Mean value } \\
& ULL & LLL & URL & MRL & LRL & \\
\hline$A_{\text {ROI }}(\%)$ & 96.89 & 95.76 & 97.12 & 97.87 & 98.17 & 97.26 \\
MEAN (mm) & 12.52 & 14.37 & 6.56 & 6.82 & 5.26 & 9.22 \\
MAX (mm) & 23.87 & 18.24 & 11.89 & 12.38 & 11.28 & 15.62 \\
SD & 0.89 & 1.10 & 0.88 & 1.03 & 0.75 & 0.93 \\
\hline
\end{tabular}

TABLE 2: Performance comparison of different segmentation algorithms.

\begin{tabular}{lccc}
\hline & Elastix & NeftyReg & $\begin{array}{c}\text { Optimal Atlas segmentation } \\
\text { algorithm }\end{array}$ \\
\hline$A_{\text {ROI }}(\%)$ & 95.21 & 94.49 & 97.12 \\
MEAN & 10.57 & 10.09 & 6.56 \\
$(\operatorname{mm})$ & & 18.65 & 11.89 \\
MAX (mm) & 17.54 & 1.48 & 0.88 \\
SD & 1.26 & &
\end{tabular}

3.2. Clinical Data of Children with MP. The comparison of the clinical data of the two groups of children is shown in Table 3. From the table data, there were 24 males and 20 females in group $\mathrm{A}$, with an average age of $4.47 \pm 1.26$ (years), and an onset time of $2.28 \pm 0.36$ (d). There were 23 males and 21 females in group B. The average age was $4.38 \pm 1.18$ (years), and the onset time was $2.32 \pm 0.25$ (d). Based on the above results, there was no considerable difference between group A and group B regarding the gender, age, and onset time $(P>0.05)$.

3.3. Detection Rate Comparison Result. CT images and $\mathrm{X}$-rays were adopted to diagnose the two groups of children with MP, and the detection results of the two groups are shown in Figures 3-5. Figures 3 and 4 showed that, in group A, 13 cases were detected in the left upper lobe, 15 cases were detected in the left lower lobe, 8 cases were detected in the 
TABLE 3: Contrast of clinical data of children with MP.

\begin{tabular}{lccc}
\hline & Group A $(n=44)$ & Group B $(n=44)$ & $P$ \\
\hline Gender (male/ & $24 / 20$ & $23 / 21$ & 0.063 \\
female) & & & \\
Age (years) & $4.47 \pm 1.26$ & $4.38 \pm 1.18$ & 0.315 \\
Onset time (d) & $2.28 \pm 0.36$ & $2.32 \pm 0.25$ & 0.326 \\
\hline
\end{tabular}

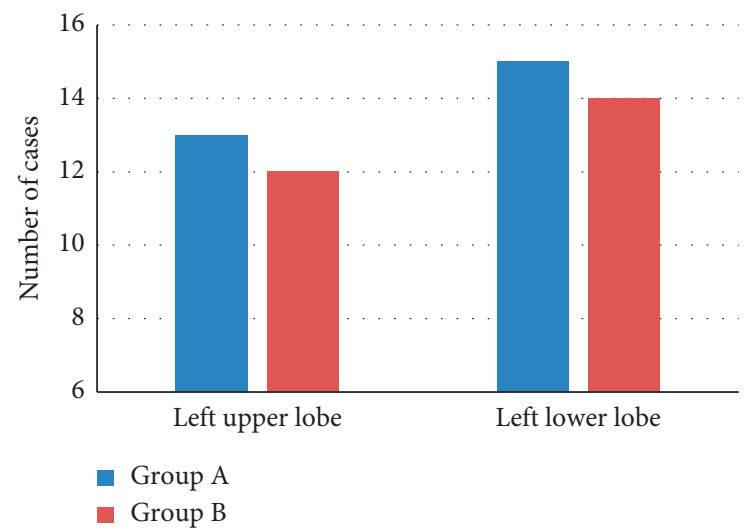

Figure 3: Contrast of left lung detection cases between two groups of children with MP.

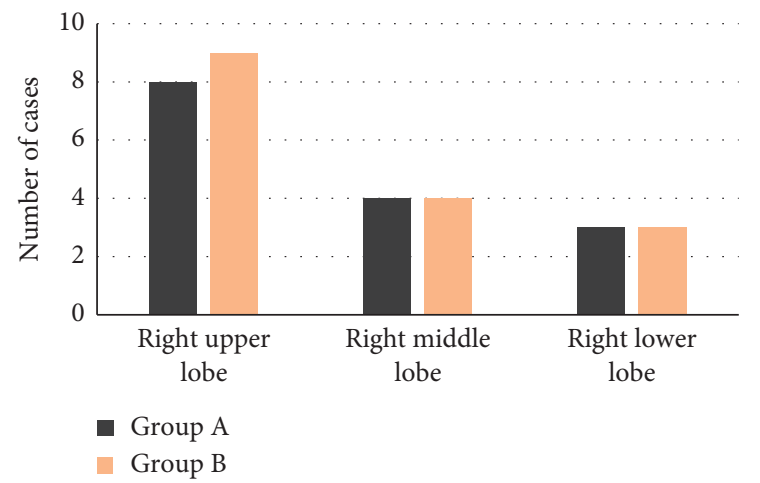

Figure 4: Contrast of right lung detection cases between two groups of children with MP.

right upper lobe, 4 cases were detected in the right middle lobe, and 3 cases were detected in the right lower lobe. In group B, 12 cases were detected in the left upper lobe, 14 cases in the left lower lobe, 9 cases in the right upper lobe, 4 cases in the right middle lobe, and 3 cases in the right lower lobe. Figure 5 disclosed that the detection rate of children in group A was $97.73 \%$, the detection rate of children in group $B$ was $95.46 \%$, and the detection rate of children with MP in groups A and B was not evidently different $(P>0.05)$.

3.4. Contrast of Imaging Features. CT examination was performed in MP children in group A, and the imaging images of some of the children are shown in Figure 6. The main features were pulmonary parenchymal shadow, largescale consolidation or patchy, pleural effusion, and so forth. $\mathrm{X}$-ray examination was performed on patients in group $\mathrm{B}$, and images of some children are shown in Figure 7, which were mainly manifested as patchy shadows, significantly increased hilar shadows, and acinar parenchyma shadows.

The imaging features of the two groups of children with MP were compared, as shown in Figures 8-10. Patchy shadows accounted for $22.05 \%$ and nodules accounted for $14.76 \%$. Acinar parenchyma shadows accounted for $26.98 \%$ and interstitial infiltration for $13.26 \%$. The proportions of increased bronchovascular shadows and pleural effusion were $9.54 \%$ and $22.03 \%$, respectively. The proportion of children in group B with patchy shadows was $24.45 \%$, and that of nodules was $12.37 \%$. Acinar parenchyma shadows accounted for $24.84 \%$ and interstitial infiltration for $13.26 \%$. The proportion of increased bronchovascular shadows was $28.32 \%$, while that of pleural effusion was $4.93 \%$. Pleural effusion detection rate in group A was greatly superior to that in group $\mathrm{B}(P<0.05)$. The detection rate of increased bronchovascular shadows in group A was inferior to that in group B considerably $(P<0.05)$. The differences of the two groups of children with nodules, patchy shadows, acinar parenchyma shadows, and interstitial infiltration were not substantial $(P>0.05)$.

3.5. Image Quality Satisfaction Comparison. The satisfaction of the attending physician with the image quality obtained by $\mathrm{CT}$ and X-ray detection is shown in Table 4 . For CT examination method, the proportions of satisfied, relatively satisfied, and dissatisfied were $74.24 \%, 24.26 \%$, and $1.50 \%$, respectively. For the X-ray examination methods, the proportions of satisfied, relatively satisfied, and dissatisfied were $65.37 \%, 14.09 \%$, and $20.54 \%$, respectively. The satisfaction with CT image quality was $98.50 \%$, which was notably higher compared to satisfaction with X-ray image quality of $79.46 \%$ $(P<0.05)$.

\section{Discussion}

Pneumonia is usually caused by a respiratory virus and occurs mostly in childhood. MP is a common cause of community-acquired respiratory infections, accounting for $40 \%$ of pneumonia cases in children above 5 years of age [12]. CT imaging technology and X-ray are clinically common MP imaging diagnostic techniques for childhood pneumonia. Due to its convenient operation, low cost, and high diagnostic accuracy, it is accepted by patients and medical workers. In this work, the OASA was adopted to improve and optimize the segmentation effect of medical CT images. The results showed that the OASA had higher accuracy for CT image segmentation results relative to Elastix and NeftyReg segmentation methods, for the index values of MEAN, MAX, $A_{\text {ROI }}$, and SD of $9.22 \mathrm{~mm}, 15.62 \mathrm{~mm}, 97.26 \%$, and 0.93 , respectively, which was consistent with the research results of Arabi and Zaidi [13], suggesting that the OASA can improve the accuracy of medical CT image segmentation.

Then, CT imaging technology and chest X-ray were adopted to diagnose children with pneumonia MP, and it was found that the detection rate of children in group A was 


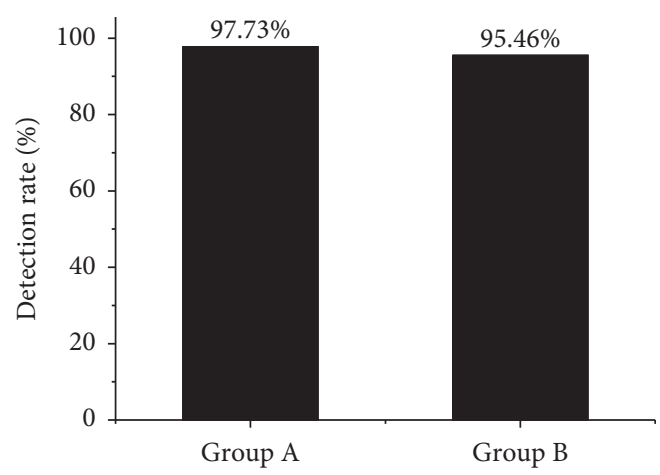

(a)

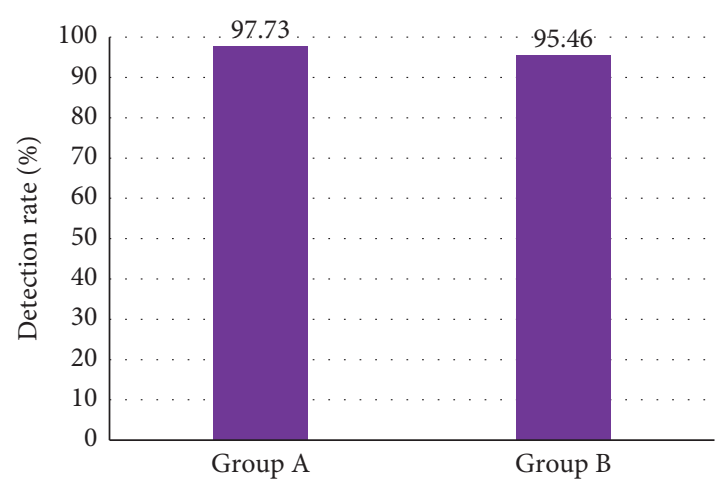

(b)

FIgURE 5: Contrast of total detection rate between two groups of children with MP.

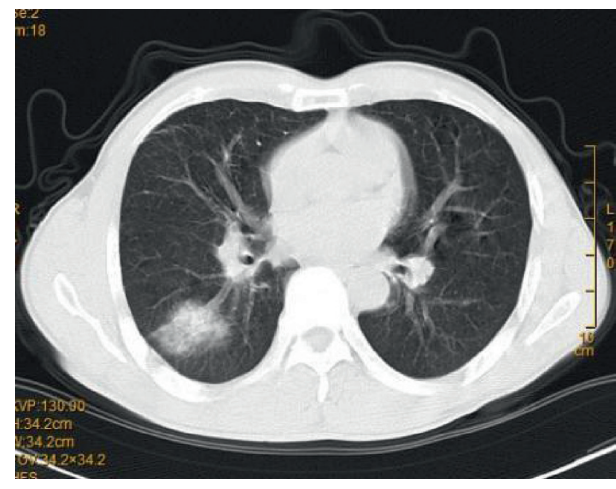

Figure 6: Chest CT image of MP children.

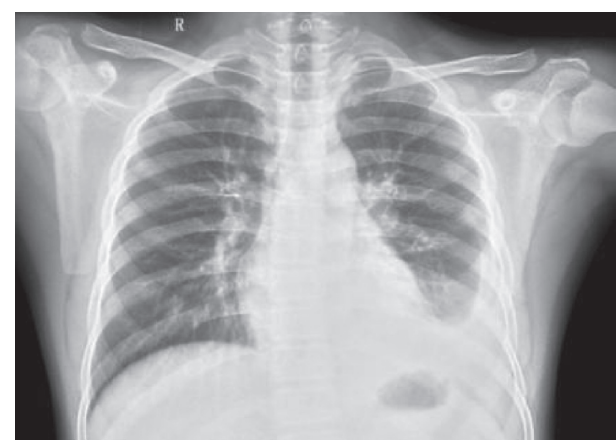

FIgURE 7: X-ray image of MP children.

$97.73 \%$, and that of children in group B was $95.46 \%$. No considerable difference was shown between the two $(P>0.05)$, indicating that both CT images and X-rays can be adopted for diagnosis of pediatric MP, and both of these imaging diagnostic techniques had high diagnostic value $[14,15]$. It was found that the main feature of CT was the shadow of the lung parenchyma, large consolidation, or patchy shadows, and pleural effusion can be seen. X-ray images were mainly characterized by increased patchy shadows, increased hilar shadows, and acinar parenchyma shadows. The pleural effusion detection rate of children in group A was evidently higher compared to X-ray group, and

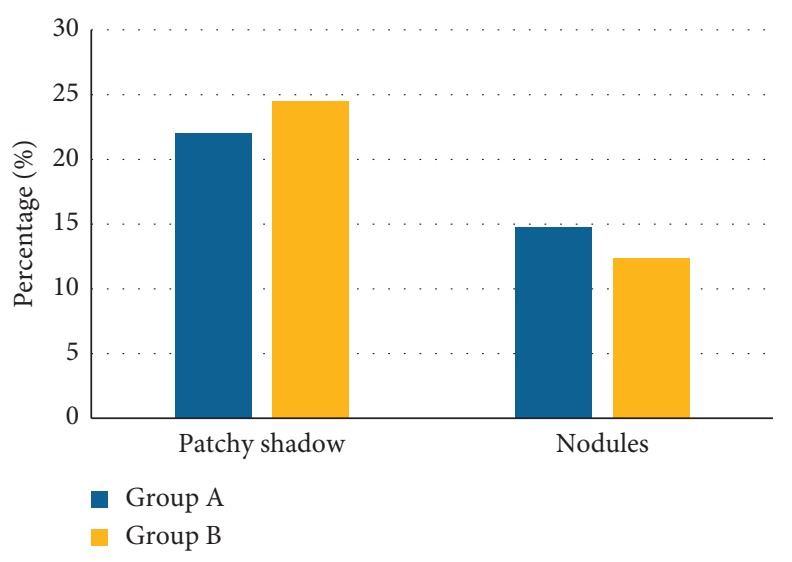

FIgURE 8: Contrast of percentage of nodules and patchy shadows between two groups of children.

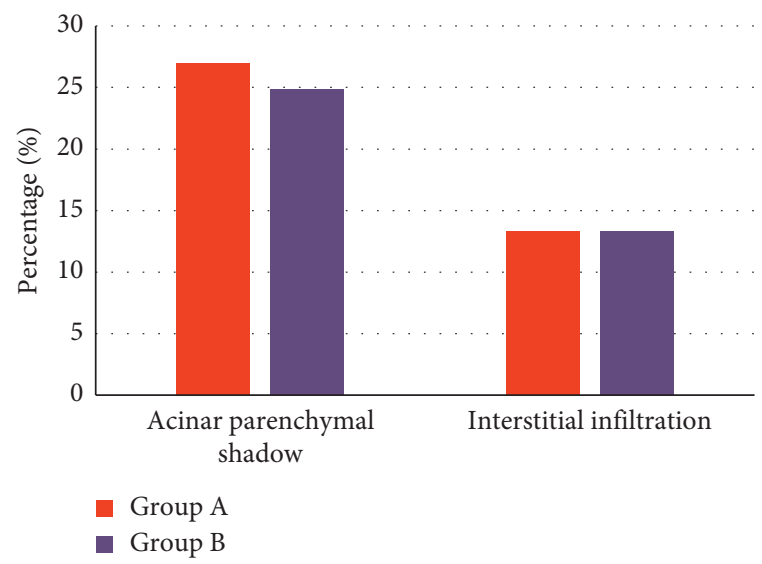

FIgURE 9: Contrast of percentage of acinar parenchyma shadows and interstitial infiltration between two groups of children.

the increased bronchovascular shadows detection rate was substantially lower versus that of the $\mathrm{X}$-ray group $(P<0.05)$. There was no obvious difference in nodule shadows, patchy shadows, acinar parenchyma shadows, and interstitial infiltration between the two groups of children $(P>0.05)$, 


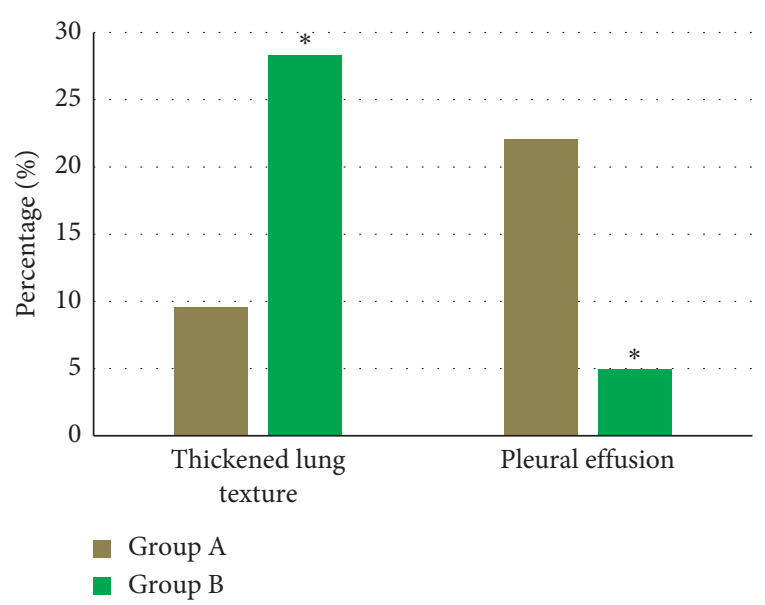

Figure 10: Contrast of percentage of increased bronchovascular shadows and pleural effusion between two groups of children $\left({ }^{*}\right.$ the differences were considerably relative to group $\mathrm{A}, P<0.05)$.

TABLE 4: Satisfaction comparison of different detection methods.

\begin{tabular}{lcccc}
\hline & CT & X-ray & $\chi^{2}$ & $P$ \\
\hline Satisfied (\%) & 74.24 & 65.37 & 0.673 & 0.282 \\
Relatively satisfied (\%) & 24.26 & 14.09 & 1.427 & 0.163 \\
Dissatisfied (\%) & 1.50 & 20.54 & 3.272 & 0.003 \\
Satisfaction rate (\%) & 98.50 & 79.46 & 4.342 & 0.001 \\
\hline
\end{tabular}

suggesting that both $\mathrm{CT}$ images and X-rays can present the specific imaging manifestations of MP, and CT was more sensitive to the diagnosis of pleural effusion, which provided a reliable basis for clinical treatment. The results were consistent with the results of Cho et al. [16] and Saraya et al. [7]. This work also revealed that the attending physician's satisfaction with CT image quality (98.50\%) was higher versus X-ray (79.46\%), $P<0.05$. Based on the above results, for children with MP, the diagnosis of CT imaging technology was more accurate. In clinical practice diagnosis, the correlation between the clinical symptoms of pneumonia MP and viral infection was high, so it was imperative to combine other laboratory indicators for comprehensive examination. In addition, both CT images and X-rays can be adopted for the diagnosis of pediatric MP, while the X-ray dose was lower, which was more suitable for children. Therefore, in the actual diagnosis, the appropriate diagnosis method should be selected according to the symptoms of the child's condition. Generally, the diagnosis can be made through X-ray and laboratory indicators, and CT examination should be taken when necessary to improve the detection rate.

\section{Conclusion}

The OASA was adopted to process the CT images and was utilized in pediatric MP clinical diagnosis. It was found that, in contrast to the traditional segmentation algorithm, the OASA enhanced the segmentation effect of CT images. Both $\mathrm{X}$-ray and CT images can diagnose MP, but CT diagnosis was more accurate and the images were clearer. However, there are still some shortcomings in the research. For example, the number of samples is limited, and there is no CT image control that is not processed by the algorithm. In the future, increasing the number of samples and using other segmentation algorithms to optimize CT images for comparative analysis of diagnostic effects should be considered. In short, the results of this study can provide a reference for the imaging diagnosis and follow-up treatment of pediatric MP.

\section{Data Availability}

The data used to support the findings of this study are available from the corresponding author upon request.

\section{Conflicts of Interest}

The authors declare no conflicts of interest.

\section{References}

[1] H. Lee, K. W. Yun, H. J. Lee, and E. H. Choi, "Antimicrobial therapy of macrolide-resistant Mycoplasma pneumoniae pneumonia in children," Expert Rev Anti Infect Ther, vol. 16, no. 1, pp. 23-34, 2018.

[2] A. K. C. Leung, A. H. C. Wong, and K. L. Hon, "Communityacquired pneumonia in children," Recent Patents on Inflammation \& Allergy Drug Discovery, vol. 12, no. 2, pp. 136-144, 2018.

[3] J. J. Xu and L. H. Shu, "[Clinical characteristics of refractory Mycoplasma pneumoniae pneumonia in children]," Zhong Guo Dang Dai Er Ke Za Zhi, vol. 20, no. 1, pp. 37-42, 2018, Chinese.

[4] X. Wang, L. J. Zhong, Z. M. Chen, Y. L. Zhou, B. Ye, and Y. Y. Zhang, "Necrotizing pneumonia caused by refractory Mycoplasma pneumonia pneumonia in children," World $J$ Pediatr, vol. 14, no. 4, pp. 344-349, 2018, Epub 2018 Jun 11. PMID: 29948966.

[5] L. I. Wijesooriya, T. Kok, J. Perera, Y. Tilakarathne, and C. N. P. Sunil, "Mycoplasma pneumoniae DNA detection and specific antibody class response in patients from two tertiary care hospitals in tropical Sri Lanka," Journal of Medical Microbiology, vol. 67, no. 9, pp. 1232-1242, 2018.

[6] L. Gong, C. L. Zhang, and Q. Zhen, "Analysis of clinical value of CT in the diagnosis of pediatric pneumonia and mycoplasma pneumonia," Experimental and Therapeutic Medicine, vol. 11, no. 4, pp. 1271-1274, 2016.

[7] T. Saraya, T. Watanabe, and Y Tsukahara, "The correlation between chest X-ray scores and the clinical findings in children and adults with mycoplasma pneumoniae pneumonia," Internal Medicine, vol. 56, no. 21, pp. 2845-2849, 2017.

[8] H. Seo, S. I. Cha, and K. M Shin, "Relationship between clinical features and computed tomographic findings in hospitalized adult patients with community-acquired pneumonia," The American Journal of the Medical Sciences, vol. 356, no. 1, pp. 30-38, 2018.

[9] F. P. An and Z. W. Liu, "Medical image segmentation algorithm based on feedback mechanism CNN," Contrast Media and Molecular Imaging, vol. 2019, Article ID 6134942, 13 pages, 2019.

[10] P. Kalshetti, M. Bundele, P Rahangdale, D. Jangra, C. Chattopadhyay, G. Harit et al., "An interactive medical image segmentation framework using iterative refinement," Computers in Biology and Medicine, vol. 83, pp. 22-33, 2017. 
[11] S. M. Lee, J. B. Seo, S. M. Lee, N. Kim, S. Y. Oh, and Y. M. Oh, "Optimal threshold of subtraction method for quantification of air-trapping on coregistered CT in COPD patients," European Radiology, vol. 26, no. 7, pp. 2184-2192, 2016.

[12] L. E. Rogozinski, B. K. Alverson, and E. A. Biondi, "Diagnosis and treatment of Mycoplasma pneumoniae in children," Minerva Pediatrica, vol. 69, no. 2, pp. 156-160, 2017.

[13] H. Arabi and H. Zaidi, "One registration multi-atlas-based pseudo-CT generation for attenuation correction in PET/ MRI," European Journal of Nuclear Medicine and Molecular Imaging, vol. 43, no. 11, pp. 2021-2035, 2016.

[14] L. Gu, W. Liu, G. X. Lai, X. Zhou, D. L. Liu, and D. S. Jia, "Oniae pneumonia," PLoS One, vol. 14, no. 8, Article ID e0219463, 2019.

[15] L. Huang, X. Huang, W. Jiang, R. Zhang, Y. Yan, and L. Huang, "Independent predictors for longer radiographic resolution in patients with refractory Mycoplasma pneumoniae pneumonia: a prospective cohort study," BMJ Open, vol. 8, no. 12, Article ID e023719, 2018.

[16] Y. J. Cho, M. S. Han, and W. S. Kim, "Correlation between chest radiographic findings and clinical features in hospitalized children with mycoplasma pneum3," PLoS One, vol. 14, no. 8,2019 . 